(c) 2018 IEEE. Personal use of this material is permitted. Permission from IEEE must be obtained for all other uses, in any current or future media, including reprinting/republishing this material for advertising or promotional purposes, creating new collective works, for resale or redistribution to servers or lists, or reuse of any copyrighted component of this work in other works. 


\title{
Simple Spread-Spectrum Pulse-Modulation Technique for EMI Mitigation in Power Converters
}

\author{
Saad Ul Hasan, Yuba Raj Kafle and Graham E. Town \\ School of Engineering \\ Macquarie University \\ NSW-2109, Australia \\ saad.hasan@students.mq.edu.au
}

\begin{abstract}
The adoption of wide-bandgap (WBG) switching devices such as gallium nitride (GaN) transistors in power converters enables to achieve higher switching frequencies and higher power density with reduced cooling requirements and smaller passive components. However, the potential for increased electromagnetic interference (EMI) resulting from the faster switching transients possible with WBG devices is a concern in many applications. Hence techniques for reducing EMI are of increasing interest. In this paper we present a simple implementation of a spread-spectrum pulse-modulation technique to reduce the peak EMI generated by switch-mode power converters. The method is based on an aperiodic gate drive signal generated from two periodic signals, e.g. a sawtooth and a sinewave, which are anharmonically related. Simulations and experimental measurements of conducted EMI from a prototype of a SEPIC converter incorporating a hybrid GaN HEMT demonstrated a $10 \mathrm{~dB}$ reduction in peak EMI.
\end{abstract}

Index Terms - Electromagnetic Interference (EMI), gallium nitride (GaN), high-electron-mobility transistor (HEMT), SEPIC, wide bandgap (WBG).

\section{INTRODUCTION}

High-frequency switching power converters are popular in applications requiring portability and compactness. Widebandgap (WBG) power switching devices, such as gallium nitride $(\mathrm{GaN})$ transistors, have attracted much attention in recent years for providing high-frequency operation along with various benefits, such as low on-state resistance and faster switching speeds, allowing the power converter to be efficient, lightweight and compact [1-2].

The integration of a renewable energy source usually requires a buck/boost operation and is realized with the help of switching power converters. Various DC-DC converter topologies are proposed which vary in accordance with the type of their applications. Power converters are reported to generate electromagnetic interference (EMI) as a common phenomenon, and it worsens with high-speed switching devices [3]. Moreover, with the increased integration of renewable energy sources, the EMI issue becomes more critical and, therefore, various electromagnetic compatibility (EMC) standards for keeping the noise emissions under specified limits are proposed, such as CISPR and IEC [4].

Traditional EMI suppression techniques include filters and shielding but, for price-sensitive portable applications, these solutions are not adopted. Various spread-spectrum techniques are reported in [5] which modify the switching-noise frequency spectrum from a train of spikes concentrated at the switching frequency and its harmonics to a smoother spectrum. The implementation methodology of these techniques defines the complexity, which increases in proportion to the number of switches in a power converter. Numerous analog and digital implementation methodologies are reported in [5-7].

\section{EMI ISSUES IN WBG-BASED POWER CONVERTERS}

All modern power converters can be viewed as a combination of power switching devices being switched in a pattern, i.e a specific frequency and duty ratio, to charge and discharge various reactive elements to produce the required voltages and currents. This common switching phenomenon leads to rapid current and voltage transitions (high di/dt and $\mathrm{dv} / \mathrm{dt}$ respectively) which are the main source of noise in a power converter. Of course, various other factors are also associated, including the circuit-board layout, soft-switching, filtering and shielding, parasitic inductance and capacitance. WBG power semiconductor devices allow high-switchingfrequency operation along with sharp switching transitions, which allows better efficiency, lower conduction and switching losses, higher power density and small size of passive filters. On the other hand, the sharp switching transients, though they reduce the switching losses involve high $\mathrm{di} / \mathrm{dt}$ and $\mathrm{dv} / \mathrm{dt}$ as compared to ordinary silicon-based switches. Hence, the EMI issue becomes more severe in WBG-based power converters. 
Fig. 1 gives a pictorial view of how the switching transients are associated with voltage and current alterations.

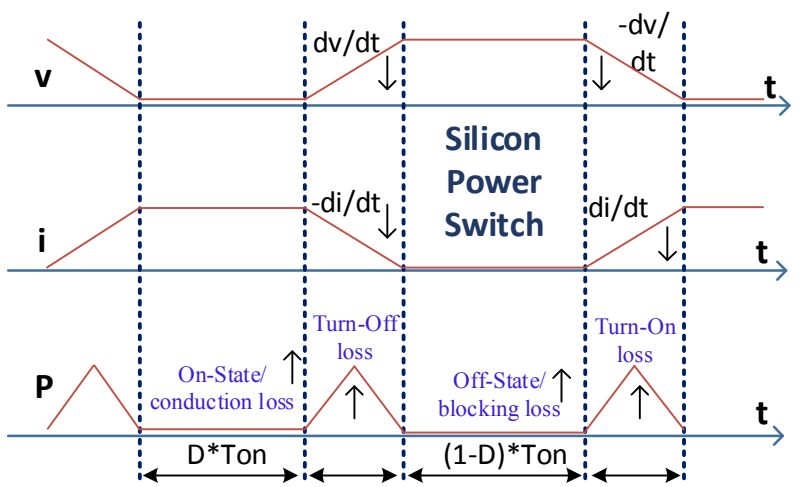

(a)

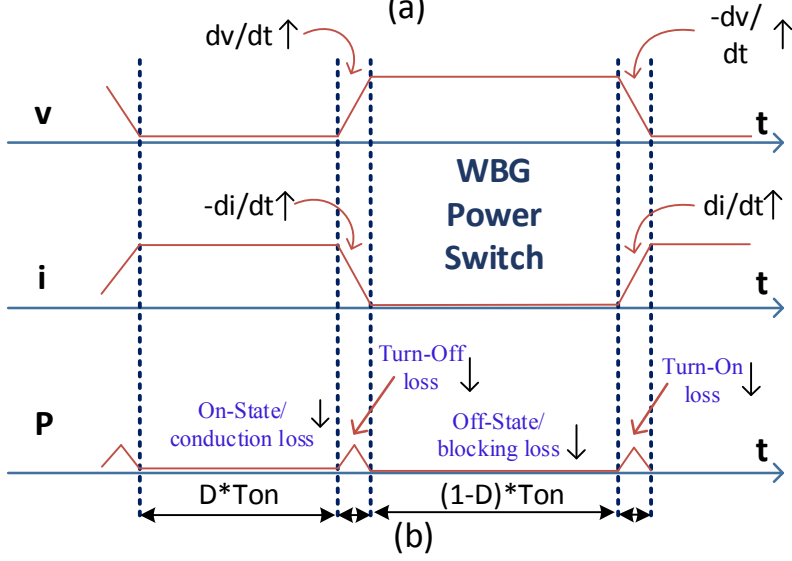

Fig. 1. Comparative overview of switching transitions between Silicon and wide-bandgap switching devices

\section{COUPLED-INDUCTOR SEPIC CONVERTER}

A coupled-inductor based SEPIC converter [8] was used for experimental investigations. The converter circuit was implemented by modifying an evaluation board from Transphorm [10] incorporating a cascode GaN (TPH3006PS) high-electron-mobility transistor (HEMT) and gate driver (FAN3100CSX). The circuit topology is shown in Fig. 2; the board modifications are shown within the dashed red line, and the design parameters were reported in [9].

\section{EMI SUPRESSION AND ITS IMPLEMENTATION METHODOLOGY}

Several techniques can be found in the literature for mitigating EMI in DC-DC converters [5-7]. Considering the PWM signal in Fig. 3 as a driving signal for a switch in a power converter, various classifications can be made based on the variation of different parameters as shown in Table. I.

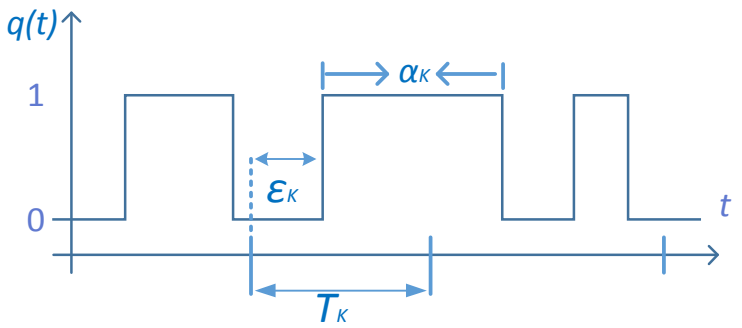

Fig. 3. A generic switching signal showing various modulation parameters

In Fig. 3, $\alpha_{K}$ is the duty cycle of a $K^{\text {th }}$ cycle having a duration defined as $T_{K} . \varepsilon_{K}$ defines the delay from the starting point of the switching cycle till the turn-on instant, hence $\varepsilon_{K}$ can be regarded as the parameter associated with pulse position. Furthermore, the switching frequency of the $K^{\text {th }}$ cycle is defined as $F_{K}=1 / T_{K}$ and duty ratio $=d_{K}=\alpha_{K} / T_{K} \cdot q(t)$ can be regarded as a switching function consisting of a series of switching cycles.

For example, of the spread-spectrum modulation techniques which can be implemented without a digital controller, one involves the use of a chaotic carrier produced by a Chua's oscillator [6]. Here we show that a simple spread-spectrum

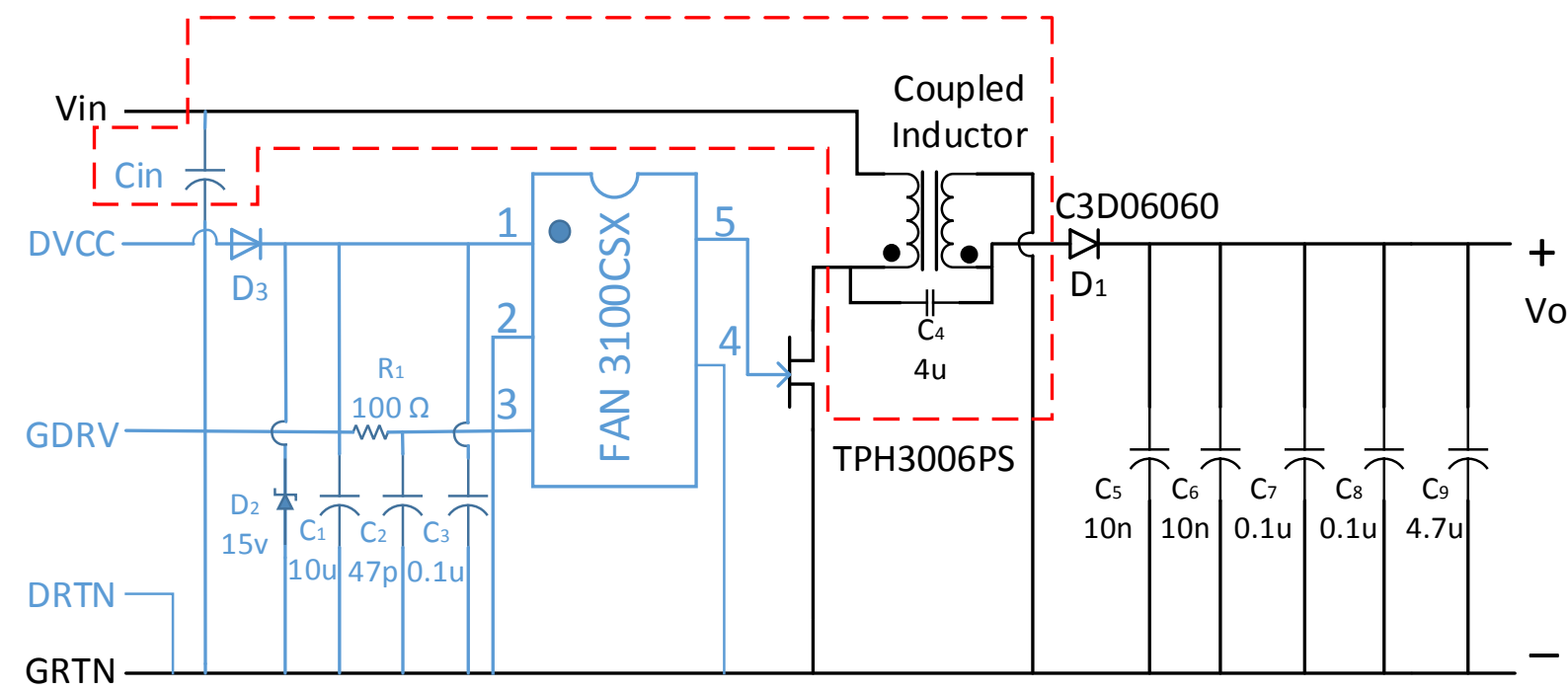

Fig. 2. Coupled-inductor SEPIC converter topology [9] 
TABLE I. Classification of spread-spectrum techniques for EMI suppression

\begin{tabular}{|c|c|c|c|c|c|c|}
\hline \multirow[b]{2}{*}{ Modulation Style } & \multirow[b]{2}{*}{ Scheme } & \multirow[b]{2}{*}{ Sub-classification } & \multicolumn{3}{|c|}{ Modulation parameters } & \multirow[b]{2}{*}{$\begin{array}{c}\text { Duty ratio } \\
\left(\frac{\varepsilon_{K}}{T_{K}}\right)\end{array}$} \\
\hline & & & $\begin{array}{c}\text { Switching } \\
\text { Cycle } \\
\left(T_{K}\right)\end{array}$ & $\begin{array}{l}\text { Duty Cycle } \\
\quad\left(\alpha_{K}\right)\end{array}$ & $\begin{array}{c}\text { Pulse } \\
\text { position } \\
\left(\varepsilon_{K}\right)\end{array}$ & \\
\hline \multirow{4}{*}{$\begin{array}{c}\text { Periodic } \\
\text { OR } \\
\text { Aperiodic (pseudo- } \\
\text { random, chaotic, } \\
\text { deterministic) }\end{array}$} & $\begin{array}{c}\text { PWM- Pulse Width } \\
\text { Modulation }\end{array}$ & -- & Fixed & Vary & Fixed & Vary \\
\hline & $\begin{array}{l}\text { PPM- Pulse Position } \\
\text { Modulation }\end{array}$ & -- & Fixed & Fixed & Vary & Fixed \\
\hline & \multirow{2}{*}{$\begin{array}{l}\text { CFM- Carrier frequency } \\
\text { modulation }\end{array}$} & $\begin{array}{l}\text { CFMFD- CFM with } \\
\text { fixed duty cycle }\end{array}$ & Vary & Sync & Fixed & Fixed \\
\hline & & $\begin{array}{l}\text { CFMVD- CFM with } \\
\text { varying duty cycle }\end{array}$ & Vary & $\begin{array}{c}\text { Fixed or Vary } \\
\text { (not synced) }\end{array}$ & Fixed & Vary \\
\hline \multirow{3}{*}{$\begin{array}{c}\text { OR } \\
\text { random }\end{array}$} & $\begin{array}{c}\text { Duty ratio modulation }+ \\
\text { PPM + fixed carrier } \\
\text { frequency }\end{array}$ & $x_{0}$ & Fixed & Vary & Vary & Vary \\
\hline & $\begin{array}{c}\text { CFM + PPM + fixed } \\
\text { duty ratio }\end{array}$ & -- & Vary & Vary (Synced) & Vary & $\begin{array}{c}\text { Fixed } \\
\text { (Synced) }\end{array}$ \\
\hline & $\begin{array}{c}\mathrm{CFM}+\mathrm{PPM}+\text { duty ratio } \\
\text { modulation }\end{array}$ & -- & Vary & Vary & Vary & Vary \\
\hline
\end{tabular}

pulse modulation can also be generated simply using anharmonically related sawtooth (carrier) and sinusoidal (modulation) signals, resulting in an aperiodically varying pulse position and pulse width in consecutive switching cycle [11]. The proposed technique is shown schematically in Fig. 4.

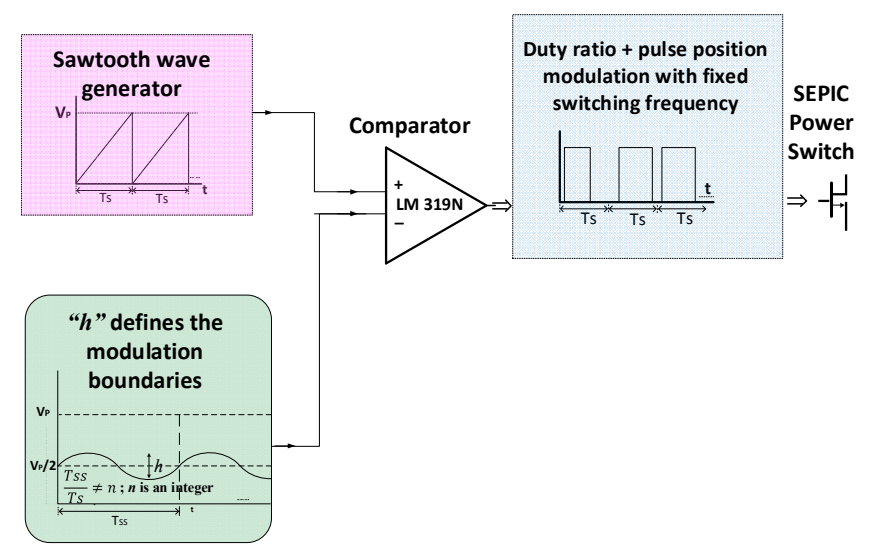

Fig. 4. Aperiodic pulse modulation generation

Both the carrier signal and the modulation signal can be simply generated by a standard function generator. The peakpeak value of the sine wave, denoted by ' $h$ ', gives the amount of variation in the duty cycle and hence the pulse position of the resulting PWM signal. It must be noted that the sawtooth and modulation signals are anharmonically related to each other, which is pictorially shown in Fig. 4 as $T_{S S} / T_{S} \neq n$, where $n$ is an integer and $T_{S}$ and $T_{S S}$ are the switching periods of the sawtooth wave and sine wave, respectively.

The FFT analysis of the reference PWM signal (fixed pulse position and duty cycle) and aperiodic PWM signal (aperiodically varying pulse position and duty cycle) which is used to drive the power switch is shown in Fig. 5, which depicts a suppressed and spreaded EMI spectrum. For simulation purposes, the frequency of sawtooth and sine wave is set at $24.4 \mathrm{kHz}$ and $9.1 \mathrm{kHz}$ respectively (i.e. anharmonically related). The number of harmonics increases with more spreading (governed by ' $h$ '), resulting in smoothing of the spectra. It must be noted that, with increasing ' $h$ ', the spreading of harmonics increases, but on the other hand it also has impact on the duty ratio of every switching cycle which in turn has a direct impact on the voltage gain of a DC-DC power converter [12].

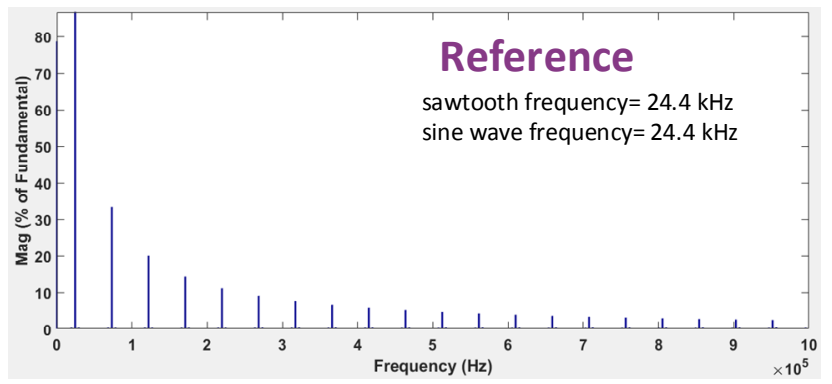

a)

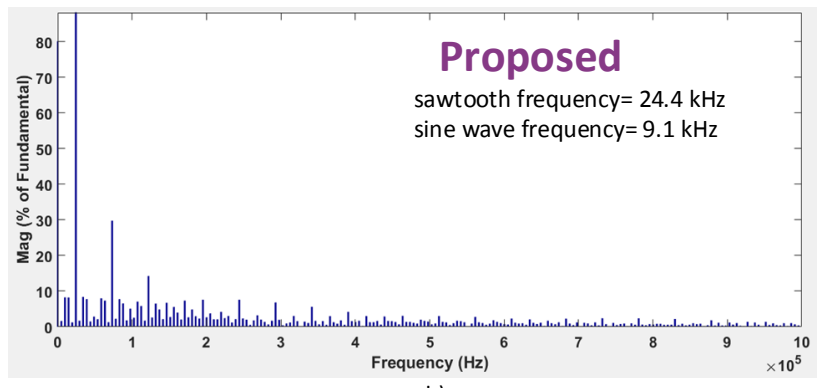

b)

Fig. 5. Fourier transforms comparing the spectra of the periodic and aperiodic pulse modulations for $10 \%$ ' $h$ '

\section{EXPERIMENTAL RESULTS AND DISCUSSION}

The PWM generated with $T_{S S} / T_{S}=1$ and $h=10 \%$ of $V_{P}$ gives a PWM with a fixed duty cycle and pulse position, whereas the PWM generated with $T_{S S} / T_{S} \neq n$ with the same 
' $h$ ' gives a varying pulse and position. The key waveforms of both the reference and the proposed modulation schemes are shown in Fig. 6. The blurred edges in the gate drive signal in Fig. 6(b) are an indication of the aperiodicity in the proposed pulse modulation scheme, which is the basis of the EMI suppression.

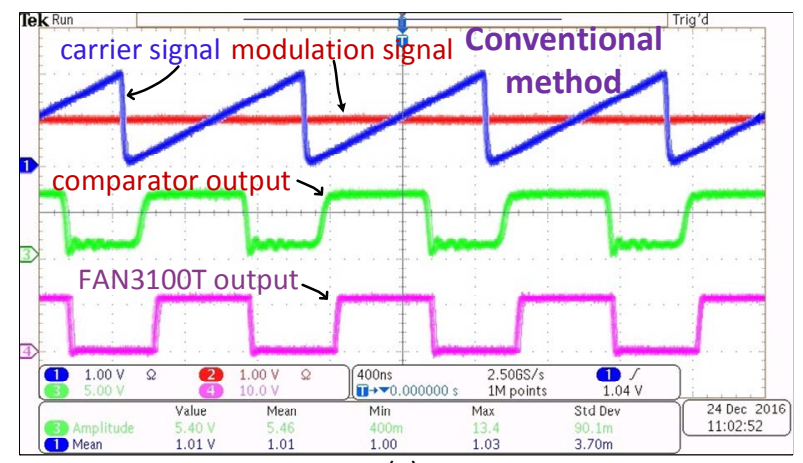

(a)

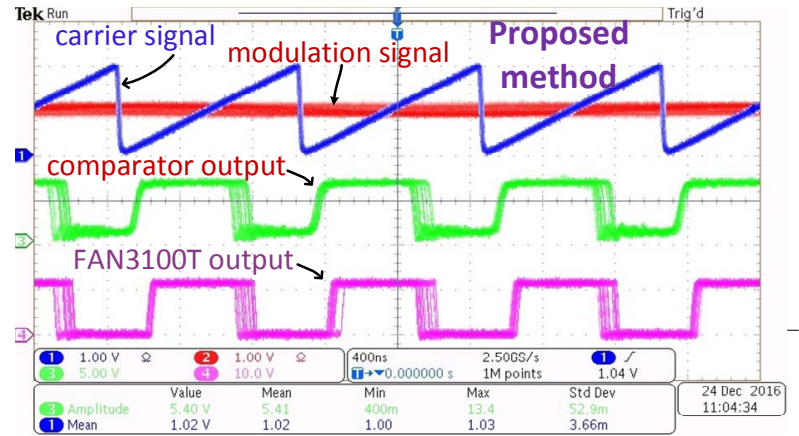

(b)

Fig. 6. Key waveforms illustrating generation of switching patterns using (a) conventional and (b) proposed method

The SEPIC converter was tested for both the reference (traditional PWM) and the proposed aperiodic modulation schemes under identical conditions for a 10 watt load with $56 \%$ duty cycle at $1 \mathrm{MHz}$ switching frequency. The sine wave frequency was $9.1 \mathrm{kHz}$ and ' $h$ ' was set to $10 \%$ of the peak of the sawtooth (carrier) wave. The conducted EMI was measured via a standard line-impedance stabilization network (LISN) [13] and the EMI spectra were plotted on a spectrum analyzer. The EMI spectra for the conventional and the proposed aperiodic modulation schemes are shown in Fig. 7. Additionally, a quasi-periodic version of the modulation scheme, similar to that reported previously [14], was generated with $n=4$, however the EMI suppression was less than with the aperiodic version [15]. This is fundamentally due to the fact that the corresponding PWM sequence will repeat itself when the frequency of sawtooth and sine wave are related to each other by an integer multiple.

\section{CONCLUSIONS}

A simple method for generating an aperiodic switching signal to reduce the peak EMI generated by single-switch power converters has been demonstrated. The aperiodic gate drive signal was generated using simple circuitry from anharmonically related sawtooth and sinusoidal signals. Experimental results with a SEPIC converter demonstrated 10 $\mathrm{dB}$ suppression in the peak EMI.

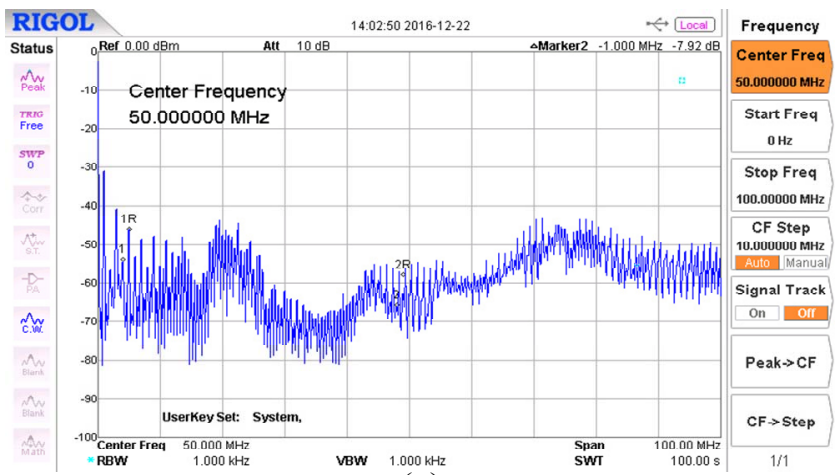

(a)

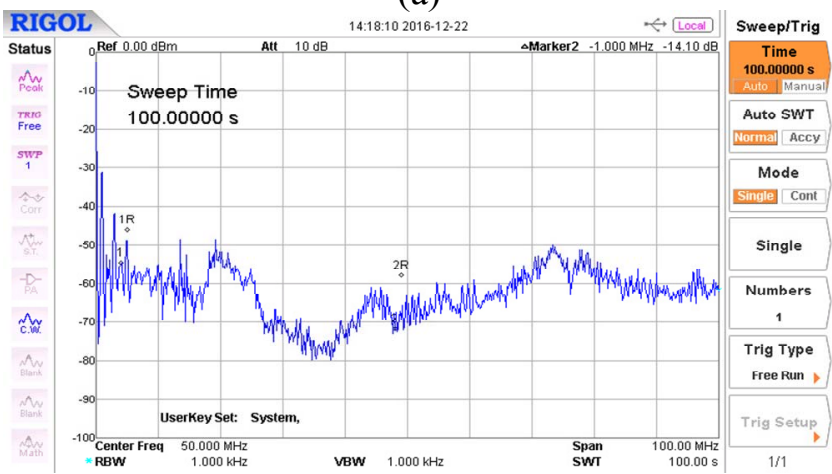

(b)

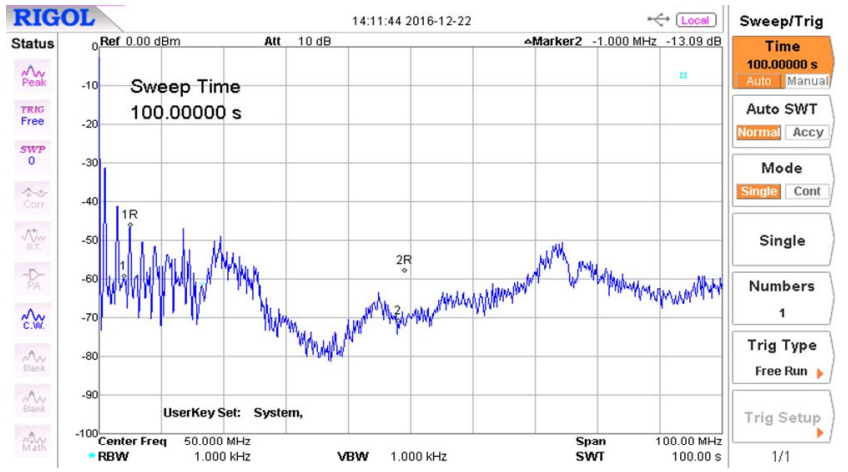

(c)

Fig. 7. Conducted EMI spectra (0-100 MHz) for a) conventional periodic, b) quasi-periodic [13], and c) aperiodic pulse modulation

\section{REFERENCES}

[1] Y. Lei, C. Barth, S. Qin, W.-C. Liu, I. Moon, S. Andrew, D. Chou, T. Foulkes, Z. Ye, Z. Liao, and R. C. Pilawa-Podgurski, "A 2 kW, SinglePhase, 7-Level, GaN Inverter with an Active Energy Buffer Achieving $216 \mathrm{~W} / \mathrm{in}^{3}$ Power Density and 97.6\% Peak Efficiency," IEEE Appl. Power Electron. Conf., 2016, pp. 1512-1519.

[2] D. Henshall, "A Pathway to Commercialization of Wide Bandgap Semiconductors in Power Electronics," Appl. Power Electron. Conf. (APEC), IS14.1, Mar. 2015. 
[3] F. Zare, D. Kumar, M. Lungeanu, and A. Andreas, "Electromagnetic interference issues of power electronics systems with wide bandgap semiconductor devices," in Proc. IEEE. Energy Conv. Congr. Expo., 2015, pp. 5946-5951.

[4] IEC. (2016), "Guidance for users of the CISPR standards". [online] Available at: http://www.iec.ch/emc/pdf/cispr_guide_2015.pdf [Accessed 30 June. 2017].

[5] G. M. Dousoky, M. Shoyama, and T. Ninomiya, "FPGA based spread spectrum schemes for conducted-noise mitigation in dc-dc power converters: Design, implementation, and experimental investigation," IEEE Trans. Ind. Electron., vol. 58, no. 2, pp. 429-435, Feb. 2011.

[6] H. Li, Z. Li, B. Zhang, F. Wang, N. Tan, and W. A. Halang, "Design of analogue chaotic PWM for EMI suppression," IEEE Trans. Electromagn. Compat., vol. 52, no. 4, pp. 1001-1007, Nov. 2010.

[7] H. Li, Y. Liu, J. Lü, T. Zheng, and X. Yu, "Suppressing EMI in power converters via chaotic SPWM control based on spectrum analysis approach," IEEE Trans. Ind. Electron., vol. 61, no. 11, pp. 6128-6137, Nov. 2014.

[8] J. Betten, "Benefit of a coupled-Inductor SEPIC converter," Texas Instruments, Dallas, TX, USA, Literature No. SLYT 411, 2011

[9] Z. Saif, V. Ahmad, G.E. Town, "Compact SEPIC converter using a GaN HEMT," Int. Conf. Power Electron. And Energy Convers. Congr. Expo. (ICPE-ECCE Asia), 2015, pp. 2249-2254.
[10] Transphorm. (2016), "Transphorm EZ-GaN Eval Board Boost Converter", [online] Available at: http://www.transphormusa.com/ sites/default/files/public/Daughter\%20Card_TDPS501E0A_0.pdf [Accessed: 30 June. 2017]

[11] S.U. Hasan and G.E. Town, "Modulation method and apparatus to reduce EMI in a power converter," AU patent WO2017120644 A1, Jan $15,2016$.

[12] K.K. Tse, H.S. Chung, S.Y.R. Hui, and H.C. So, "A comparative investigation on the use of random modulation schemes for DC/DC converters," IEEE Trans. Ind. Electron., vol. 47, no. 2, pp. 253-263, Apr. 2000.

[13] Tekbox, " $5 \mu \mathrm{H}$ Line Impedance Stabilization Network LISN" [Online]. Available:https://www.tekbox.net/images/documents/openhardware/tbo h01/TBOH01_Manual.pdf [Accessed: 30 June. 2017]

[14] S.U. Hasan and G. Town, "A quasi-periodic modulation strategy to mitigate EMI for a GaN-based Quasi-Z-Source DC-DC converter", in Proc. IEEE. Energy Conversion Congress and Expo (ECCE), pp. 1-5, Milwaukee, WI, USA, Sept, 2016.

[15] S.U. Hasan and G. Town, "An FPGA-based Aperiodic Modulation Strategy for EMI Suppression in Quasi-Z-Source DC-DC Converters", in Proc. IEEE. Int. Symp. Circuits \& Syst. (ISCAS), pp. 1-4, Baltimore, USA, May, 2017. 